\title{
RELATION EXTRACTION FOR PROTEIN-PROTEIN INTERACTIONS AFFECTED BY MUTATIONS
}

\author{
A Thesis \\ submitted to the Faculty of the \\ Graduate School of Arts and Sciences \\ of Georgetown University \\ in partial fulfillment of the requirements for the \\ degree of \\ Master of Science \\ in Computer Science
}

By

Ziling Fan, M.S.

Washington, DC

April 7, 2018 
Copyright (C) 2018 by Ziling Fan All Rights Reserved 


\title{
RELATION EXTRACTION FOR PROTEIN-PROTEIN INTERACTIONS AFFECTED BY MUTATIONS
}

\author{
Ziling Fan, M.S.
}

Thesis Advisor: Nazli Goharian, Ph.D.

\begin{abstract}
Precision Medicine (PM) is a promising approach for cancer treatment in the modern medical practice. Information about protein-protein interaction and mutations affecting the interaction is essential in understanding biological processes and is one of the key aims in PM research. While previous research in text mining has achieved great progress in extracting protein-protein interactions (PPIs) in biomedical literature, few efforts have been made to explore methods to extract PPIs which are affected by mutations.

In this thesis, I propose a feature-rich supervised method to extract PPIs affected by mutations from biomedical literature. First, a supervised model is trained to predict if a pair of proteins is interacting for new instances. Next, a 'mutation refinement' step is incorporated as a filter to determine the final answer. I compared effectiveness of two different training corpora (i. BioCreative VI PM track training; ii. AIMed+BioInfer) for model training. Experimental result shows that supervised model trained with combined corpus (AIMed+BioInfer) achieved better performance. Additionally, features selected from previous PPI extraction work and additional features were tested for model training. Evaluation of the result using BioCreative VI PM track testing dataset proves the effectiveness of the features proposed in my method. The system achieves up to $44 \%$ to improvement in F1-score over baseline method.
\end{abstract}




\section{ACKNOWLEDGEMENTS}

I would like to express my deepest thanks to Nazli. Thank her for leading me to do this research project; thank her for taking large amount of time and energy for instructing me, helping me reviewing and revising the thesis. I've really learnt a lot from her. This memorable project experience really made this degree special and I will never forget it.

I would like to say thanks to Luca, my Ph.D. student supervisor. He has given me great help over the whole process of the thesis research, giving me suggestions for project, helping me on the paper, helping me preparing for defense presentation. After first dry-run for presentation, his comments were even longer than my slides...

I would like to say thanks to the rest of my committee, Ophir and You-shin, for their valuable and insightful comments for the thesis.

I would like to say thanks to my two program directors, Lisa and Jeremy. Without Lisa, all of these in last two years may never happen. Jeremy gave me lots of encouragement when I was in hard time and I really enjoyed his algorithm lectures.

I would like to say thanks to my families who are always there when I need them. Love you guys. 
I would like to say thanks to my friends who are here in Georgetown, in US and in China. You guys are always important to me in my life, I would like to present your names here: Lan Yu, Dian Wang, Xiaoxuan Li, Wenjie Song, Quan Sun, Shuo Wang, Wei Liu. 


\section{TABLE OF CONTENTS}

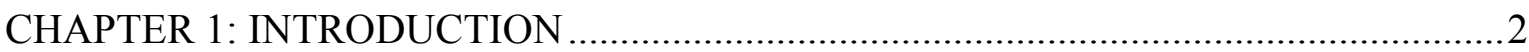

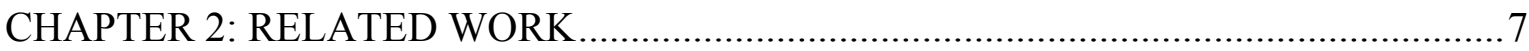

2.1 Protein Name Recognition ..................................................................................

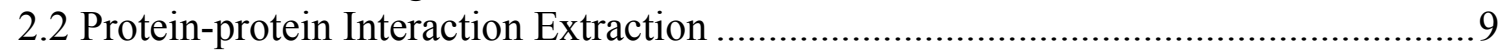

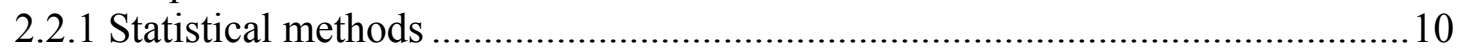

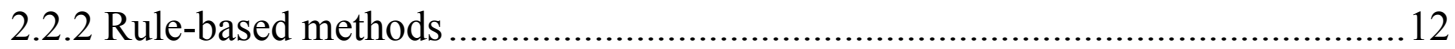

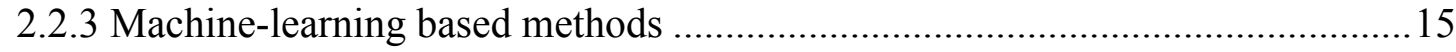

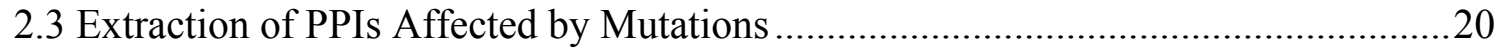

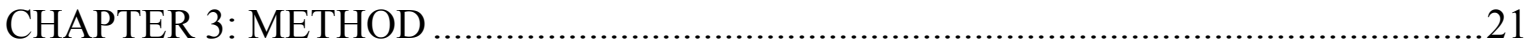

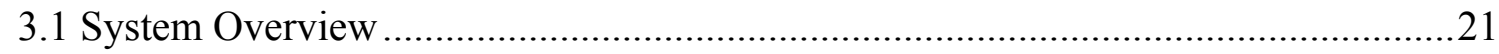

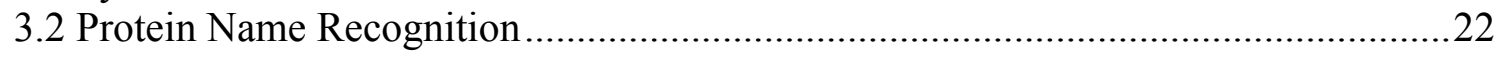

3.3 Candidate Protein Pair Extraction......................................................................23

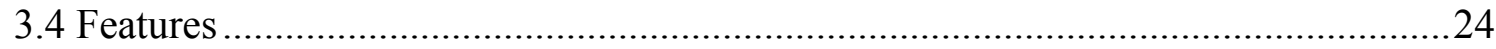

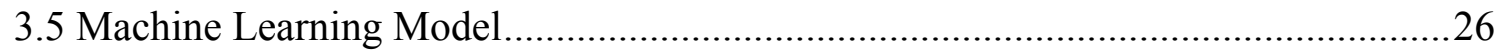

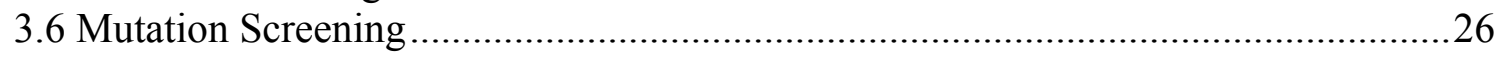

CHAPTER 4: EXPERIMENTATION RESULTS AND ANALYSES ............................28

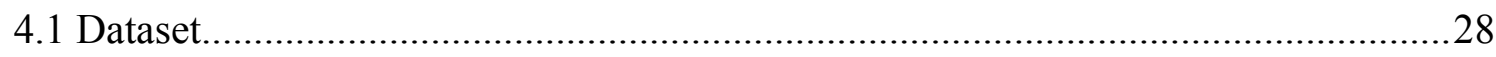

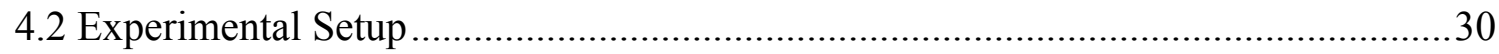

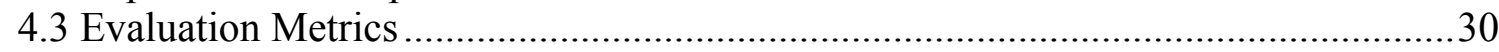

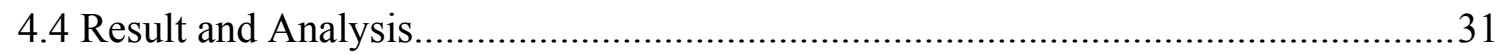

4.4.1 Comparison with existing systems...................................................................

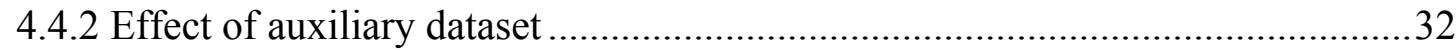

4.4.3 Effect of negation detection feature and shortest path feature.............................34

4.5 Error Analysis on False Positives and False Negatives …………...............................34

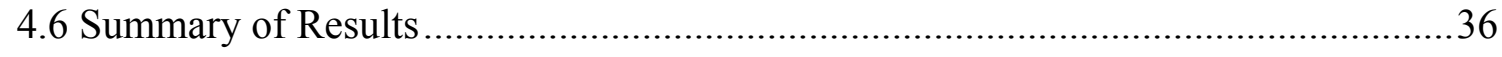

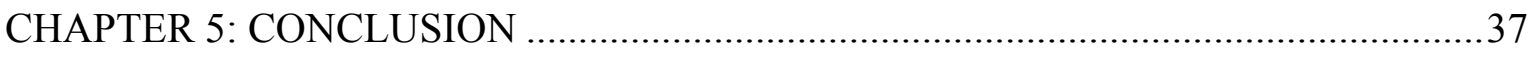

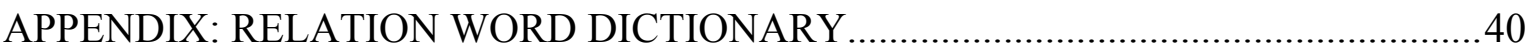

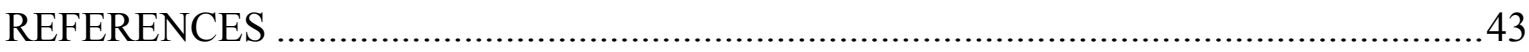




\section{LIST OF FIGURES}

Figure 1. The process of co-occurrence method incorporated with 'Relation Word

Dictionary' checking

Figure 2. Algorithm to extract PPIs from complex sentences containing more than two protein entities [5]

Figure 3. Overview of the CNN model proposed by [22] ..............................................18

Figure 4. Dependency graph for the example sentence.................................................... 18

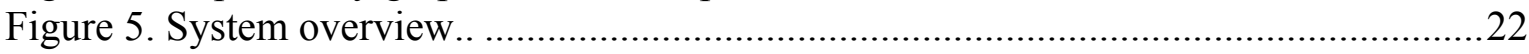

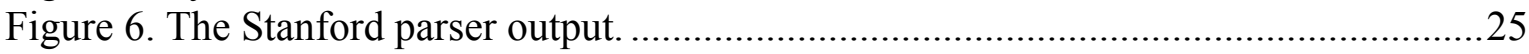

Figure 7. Dependency graph for the example sentence....................................................25

Figure 8. A snapshot of visualization of BioCreative VI PM Track

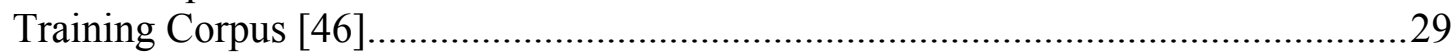

Figure 9. A snapshot of converted BioInfer corpus............................................................30

\section{LIST OF TABLES}

Table 1. Three abstract forms of expression of PPIs in biomedical literature [5] ...............14

Table 2. Statistics of BioCreativeVI PM Track training and testing corpus. .......................28

Table 3. Statistics of AIMed and BioInfer corpora...........................................................29

Table 4. Evaluation results for my method and participating teams in BioCreative VI PM

Track [42]

Table 5. Comparison of the effectiveness of system trained by different training corpus. .33

Table 6. Comparison of the effectiveness of added features used for classifier training. ...34 


\section{CHAPTER 1: INTRODUCTION}

Nowadays, in the area of tumor biology and cancer treatment, precision medicine (PM) is one of the hottest topics and has become increasingly promising as the development of next generation sequencing (NGS) [1]. According to Precision Medicine Initiative, 'PM aims to identify individualized treatment depending on a patient's genetic profile and related responses.' In terms of cancer, one of the most significant factors affecting personal choice of treatment is the mutation carried by a protein molecule from an individual patient [2]. Amino Acids serve as building blocks for protein molecules which further are the basic units of living creatures. Functional protein molecules need to have the correct sequence of amino acids to maintain an appropriate 3D structure and to carry out biological functions by interacting with other proteins. Mutations in a protein refer to the change of a correct amino acid to another one. This alteration may lead to failure of proper protein interaction and cancer may arise from the malfunction of this inevitable biological process. What role dose protein mutations play in PM? For example, two liver cancer patients with similar symptoms may bear totally different protein mutations: patient A's cancer may be due to a mutation of protein red and patient B's cancer may be due to a mutation of protein black. Without knowing the exact reason (mutation) of the disease, it's hard for doctors to give the most effective treatment to patients. On the contrary, if doctors possess mutation information of patients and have a comprehensive understanding of how mutations cause the disease, this 
will allow them to predict more accurately which treatment and prevention strategies for a particular disease will work in specific groups of people. It is in contrast to a one-size-fitsall approach, in which disease treatment and prevention strategies are developed for the average person, with less consideration for the difference between individuals. Hence, as an essential step towards the goal of PM, biomedical researchers have put a great amount of effort into studying protein-protein interactions (PPIs) and on studying which specific mutated amino acid interferes with proper interaction between two proteins.

Over the last decade, with the fast development of modern biological experimental techniques, the speed of production of research results regarding to PPIs and corresponding mutation information has improved greatly. As a result, an incredible wealth of research articles describing new findings of PPIs and mutations has been produced and stored in biomedical literature. The accumulation of these valuable research findings help us to understand the disease mechanisms at a systems level [3]. Recently, several relevant PPIs databases have been created, such as BIND, HDPR and IntAct as tools for organized and systematic data storage, data management and data retrieval. Biomedical researchers have just begun to submit their experiment results directly to these databases as a step required by publishers to ensure quality [4].

However, there is still large amounts of previously published articles which contains information of PPIs not yet curated and stored into databases and thus difficult to be fully utilized by researchers. People have tried to do information extraction and curation manually and it has been proved to be a time and resource consuming task. It becomes clear that 
automatic approaches to extract PPIs which are affected by mutations from biomedical articles is desired and will be a huge aid to the goals of Precision Medicine.

In the text of biomedical articles, the meaning of 'two proteins interact' can be expressed in a wide range of ways. The most straightforward way is 'protein1 interacts with protein2'. Similarly, the key relation word 'interact(s)' can be replaced with a noun phrase and the sentence transformed into 'interaction between protein1 and protein2'. As such, there are other nouns or verbs that convey the meaning of 'interaction', such as 'bind', 'connect', 'activate'. Even some specific biological processes indirectly indicate there must be interactions between two proteins, such as 'phosphorylation (refers to a transfer of phosphate groups between two proteins)', glycosylation (transfer of glycol group) and so on. In order to properly deal with these situations, the system requires the construction of a comprehensive relation words dictionary when doing information extraction to achieve the fullest coverage. Another situation which makes PPIs extraction harder is when there are multiple protein mentions (more than two protein entities) in a sentence. For example, our analysis reveals that protein1 form a close bounding with pro2 instead of pro3. Several methods have been proposed to break down and analyze this complex sentence grammar for the purpose of relation extraction such as rule-based extraction, constituency parsing, and they will be reviewed in the next chapter. It is worth mentioning some seemingly small details may have a significant influence on the system performance. For example, negation words, such as not, none, cannot, in front of relations word indicates that two proteins cannot interact. The extraction system needs to take this situation into consideration. 
Furthermore, extracting not only PPIs but also mutations which interferes with proper interaction make even higher demands of the system. The following example helps to understand this task better. 'We show that prol cannot bind to mutated pro2' should be selected as positive by the system because this sentence meets the two requirements of the task: 1) There is a mentioning of interaction of two proteins; 2) the mutation of pro2 abolishes this interaction. If there is no 'mutated' in the sentence, this sentence should be judged as negative. If the system cannot deal with this properly, this type of sentence may be mistakenly classified to positive and becomes a false positive.

In biomedical natural language processing (NLP), there are two research focuses in solving this task: 'PPIs extraction' and 'mutation detection'. Several methods have been proposed to address the task of PPIs extraction and they can mainly be divided into two main categories, rule-based methods and machine learning (ML) methods. Rule based methods start with defining a set of rules to extract candidate protein pairs and some specific patterns are applied to determine the final interacting protein pairs [5]. ML-based methods manage to combine NLP technique to train ML models for classification tasks on new instances [69]. Besides extraction of PPI from literature, researchers have attained great success in the task of extracting protein mutations from biomedical articles [10-12]. Wei et al. developed a text mining tool called tmVar using conditional random field (CRF) and achieved state of the art performance (90\% in F-measure) [16]. However, how to combine these efforts to identify and extract from the biomedical literature protein-protein interactions affected by mutations is a new task and very few attempts have been made into this task. 
In this thesis, I propose a feature-rich supervised method to accomplish this task. First, an ML-model is trained with a wide range of features (part-of-speech, dependency, semantic, negation etc.) and used to predict if a pair of proteins is interacting for new instances. Next, a 'mutation screening' step is incorporated as a filter to determine the final answer. To assess the system performance, I use latest BioCreative VI Precision Medicine datasets and the result demonstrates the effectiveness of my method for accomplishing the task.

The thesis is organized as follows: Chapter 2 introduces related work including protein name recognition, PPIs extraction and latest attempts of PPIs extraction with mutations. Chapter 3 discusses my approach to solve the task. Experiments using Biocreative VI datasets and evaluation results will be discussed in chapter 4. Conclusion and future work will be presented in Chapter 5. 


\section{CHAPTER 2: RELATED WORK}

There are two main steps to accomplish PPIs extraction task: protein name recognition and PPI extraction. This chapter will first give a general overview of methods proposed for protein name recognition and the focus of this chapter will be a comprehensive review of PPIs extraction approaches. Finally, although the task of extraction of PPIs affected by mutations is a relatively new area of research in this domain, and limited attempts have been made, several valuable pilot exploratory studies have been carried out and will be discussed in this chapter.

\subsection{Protein Name Recognition}

Like Named-entity recognition (NER) in any relation extraction tasks, protein name recognition paves the way for PPIs extraction and recognition performance has a huge impact to PPIs extraction because of its upstream position [10]. Although in some domains NER has achieved satisfactory performance, protein recognition in biomedical articles remains a challenging problem. Two main challenges for this task are language variation and ambiguity. Orthographical variation is one type of language variation, for example, 'p53' and ' $p-53$ '; abbreviation also leads to this issue, for example, cox 2 transcriptional factor and cox2 TF. Language ambiguity, firstly, comes from the fact that proteins from different species may share the same name, however, they are totally different proteins (TGF-beta can be either human protein or mouse protein). Second, different proteins sharing the same name adds to the ambiguity. For example, 'jun proto-oncogene' and 'FBJ murine osteosarcoma viral oncogene homolog" have a common name, 'AP-1', which is hard for automatic extraction tools. 
Takagi et al. [11] and Coster [12] tried to accomplish this task using hand-crafted rule-based method. After a close analysis of protein name lexicon, they summarized a group of feature terms (e.g. phrases containing receptor, enzyme, ligand etc.) and core terms (words ending in -ase and -in) and considered phrases in sentences rendering these features are names of proteins. Also, they used a dependency parser to determine potential locations of protein names, hence reducing the search space. However, all rule-based methods suffer from a low recall rate.

More advanced automated recognition system takes advantage of machine learning methods such as Conditional Random Field (CRF) [13] and Support Vector Machine (SVM) [10] and achieves better performance compared to rule-based extraction methods. Mitsumori et al. [10] tried to solve the problem using SVM classifier trained with both internal source features (part-of-speed, orthography, prefix, suffix and preceding class) and external features, i.e. features of matching against a protein name lexicon. Wei et al. [13] developed a comprehensive system called GNormPlus. This system not only has protein recognition function but also outputs the gene id of protein names detected in the text. In protein recognition module, they chose to adapt CRF model which is a probability based learning model and can be used to solve sequence labeling tasks. Features selected to train the model includes: 1) general linguistic features (eg. original token, stemmed token, pos, prefixes and suffixes et.); 2) character features (eg. uppercases, lowercases, letters, digits and special characters); 3) semantic features; 4) case pattern features and 5) contextual features. Currently, GNormPlus has start-of-the-art performance with $86.7 \%$ F1-score on the 
BioCreative II gene Normalization task dataset and $50.1 \%$ F1 score on the BioCreative III gene Normalization task ${ }^{1}$.

\subsection{Protein-protein Interaction Extraction}

PPIs extraction efforts have included concepts and techniques including Machine Learning (ML), Natural Language Processing (NLP) and Information Extraction (IE) [14-17]. Earlier attempts adopted simpler statistical methods, such as co-occurrences, and pattern matching methods $[18,19]$; By the development of more advanced linguistic tools and deeper understanding of the task of PPIs extraction, researchers have been exploring more complex semantic and syntactic features of biomedical literature text and more advanced algorithm methods, such as ML and deep learning methods [20-22]. This section presents a survey of methodologies proposed to accomplish this task.

Although most research efforts adopt a mix of techniques, they can generally be divided into three main categories:

- Statistical methods. The number of occurrence of protein entities in a 'block of text' is the most straightforward statistical feature for PPIs extraction task. Taking advantage of occurrence of two mentions in a relation is a common baseline method for any relation extraction task. Co-occurrence is one of the most commonly used features in PPIs extraction task [19]. For example, PRO1 shares the same ATPbinding domain with PRO2. Co-occurrence method will assume any pair of protein

\footnotetext{
${ }^{1}$ http://www.biocreative.org/resources/corpora/biocreative-iii-corpus/
} 
entities in the same sentence render positive relation. Therefore, in the sentence above, $P R O 1$ and $P R O 2$ are considered as interacting.

- Rule-based methods. Although text is seemingly unstructured, the way to express two proteins interact is relatively fixed. The rule-based method is to try to summarize all possible ways of expression as rules through large amount of close observation and extract textual relationships based on these rules.

- Machine learning methods. In contrast to the rule-based method in which a handcrafted rule set is created manually for the purpose of extraction in unstructured text, ML-based methods work via training models to recognize features of positive against negative relations using labeled data (two proteins interact is a priori) and make decisions for unlabeled new data.

\subsubsection{Statistical methods}

The most common statistics used in relation extraction is co-occurrence [19]. As in real life, any two events happening in a timeframe may be related while those that happen in different timeframes are not related. Similarly, we can think of two entities appearing in a "block of text' as holding a semantic relation. Typically, a block can be a phrase, a sentence or a paragraph. In some special circumstances, a larger building block may be selected as a unit such as a section or even a chapter. For the purpose of PPI extraction, sentence is the most optimal building block [17]. However, the semantic relation between two co-occuring entities of co-occurrence is not necessarily 'interaction'. Pyysalo et al. [23] made a comparative analysis for five protein-protein interaction corpora and pointed out that only $17 \%$ of sentence-level protein entities render the meaning of interaction. Consequently, without taking any semantics or syntactic information into consideration, solely depending 
on co-occurrence barely gives a satisfactory performance. However, incorporation of additional filters helps to improve the performance, such as using co-occurrence of entity pairs in larger block (i.e. paragraph, passage) as the second threshold or requiring a key word (indicates 'interaction') in the sentence besides the co-occurrence of the entity pair [24]. Although [24] is still a relatively simple method, it achieves much better performance compared to pure co-occurrence baseline method and even a comparable performance with more complex methods for some datasets. In the next section, the method proposed in [14] will be illustrated as an example of statistical method.

\subsubsection{An example}

As Figure1 demonstrates, after protein annotation, the same pair of protein entities are recognized both in (a) and (b). Based on the idea of co-occurrence, because 'megalin' and 'NHERF1' occurs in the same sentence in both sentences, both pairs can be thought as positive PPI and extracted as candidates. However, in the step of 'interaction word dictionary' check, 'bound' in the sentence (a) is found in the dictionary but no interaction word is found in sentence (b). Therefore, the output of this algorithm for sentence (a) is the annotated protein pair and for sentence (b) is None. 


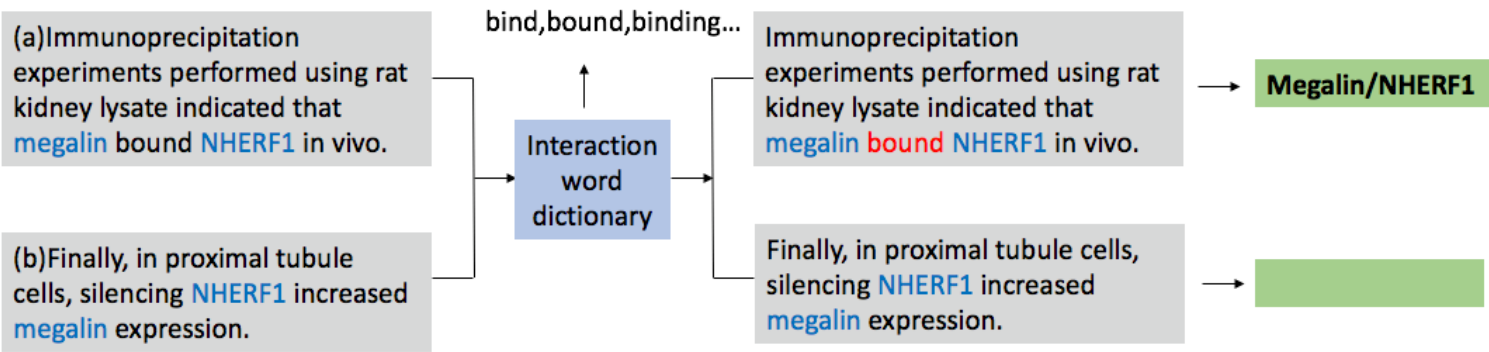

Figure 1. The process of co-occurrence method incorporated with 'Relation Word Dictionary' checking. Any two protein mentions existing in one sentence are considered as candidate output. After relation dictionary screening, only the protein pair which is in a sentence containing a relation word is output.

\subsubsection{Rule-based methods}

Rule-based methods are also called pattern matching methods. SUISEKI (System for Information Extraction on Interactions) is one of the first attempts to extract PPIs in biomedical literature using hand-crafted rules and those rules are called frames [25]. They showed that one of the frames which has the highest matching is the '[protein/gene] binds/associates/...[protein/gene]'. A distance measure between two entities is also incorporated in the system to assign a probability score to each potential entity pair. Overall, around 40 such patterns were manually created which helps to achieve a satisfactory precision. However, low recall is inevitable due to high missing rate caused by limited patterns.

Apart from contextual information, NLP parsers are utilized to analyze sentence patterns which then are used to extract PPIs from text. Toshihisa [26] explores syntactic patterns in biomedical articles and uses them as rules to extract PPIs. They also consider negation expression in sentences. With pre-annotated (protein names) data, it achieves a good performance for Saccharomyces cerevisiae(yeast) and Escherichia coli dataset with an 85\% 
recall rate and $84 \%$ precision rate. In BioCreative II, [27] proposed a system called OpenDMAP which is an ontology-driven template matching framework with an average F1 score of 0.29 and is the best system in BioCreative $\mathrm{II}^{2}$.

To have a comprehensive coverage of patterns and avoid laborious pattern craft, Huang et al. proposed an automatic pattern generation method [28]. In pattern generation phase, they started with tagging sentences with part-of-speech tags and replace protein names with PTN. They also associate a word set with each pos tag; for example, in pattern \{PTN VBZ IN PTN\}, the VBZ has to be words indicating the meaning of interaction, such as 'binds', 'associates' or 'interacts'. Next, dynamic programming is used to extract all of the patterns in training corpus. [29] adopted a similar method for BioCreative II and the evaluation result shows that this method achieves an F1 score of $24 \%$ without any corpus-specific tuning. The author concludes that this approach requires a high consistency between training dataset and testing dataset otherwise patterns generated from the training set hardly give a satisfactory matching rate.

\subsubsection{An example}

In this section, I illustrate the process of adapting a rule-based method to extract PPIs in biomedical literature with a specific example, PPInterFinder, which is developed by Natarajan and coworkers [5].

\section{i. Abstract forms for PPI candidate pairs}

After close observation of biomedical literature, they summarized 3 types of abstract forms commonly used by authors to express interaction between two protein entities as

\footnotetext{
${ }^{2}$ http://www.biocreative.org/resources/corpora/biocreative-iii-corpus/
} 
demonstrated in Table 1 The most primary difference over these three groups are the relative position of relation words.

Table 1. Three abstract forms of expression of PPIs in biomedical literature [5].

\begin{tabular}{ll}
\hline Form1 & PROTEIN1 - token* - RELATION - token* - PROTEIN2 \\
\hline Example & $\begin{array}{l}\text { PROTEIN1 interacts with PROTEIN2 } \\
\text { PROTEIN1 has weak association with PROTEIN2 }\end{array}$ \\
\hline Form2 & RELATION - token* - PROTEIN1 - token* - PROTEIN2 \\
\hline Example & interaction between PROTEIN1 and PROTEIN2 \\
\hline Form3 & PROTEIN1 - token* - PROTEIN2 - token* - RELATION \\
\hline Example & PROTEIN1 and PROTEIN2 complex \\
\hline
\end{tabular}

\section{ii. $\quad$ Rule set for identification and extraction of candidate PPI pairs}

In the second step, they set up rules for three abstract forms respectively. For example, one of the rules is the distance between the protein pairs. Only protein pairs within a certain distance would be extracted. It's worthwhile to mention that these three abstract forms actually can cover more complex sentences containing more than two protein names. For example, in form 1, there may be two proteins before relation word and one after relation word. In the rule set they created, there are some rules designed specifically for these complicated situations. Figure 2 shows the procedure for extracting PPIs from sentences containing more than two protein names. After protein recognition and relation words/negation words are detected, the algorithm starts with extracting candidate PPIs based on the predefined rule set. In our example, three groups of candidate PPIs are generated based on the original order of the sentence and combing the relation word with each of preceding and succeeding protein names. Finally, in order to have a good control of false 
positive rate, a pattern dictionary was created to accomplish the final filtering step. We can see in the example only the first two entries are kept because the third entry which is in the pattern of $\{$ pro + Rel + neg +$\}$, not in the pattern dictionary, is excluded in final output.

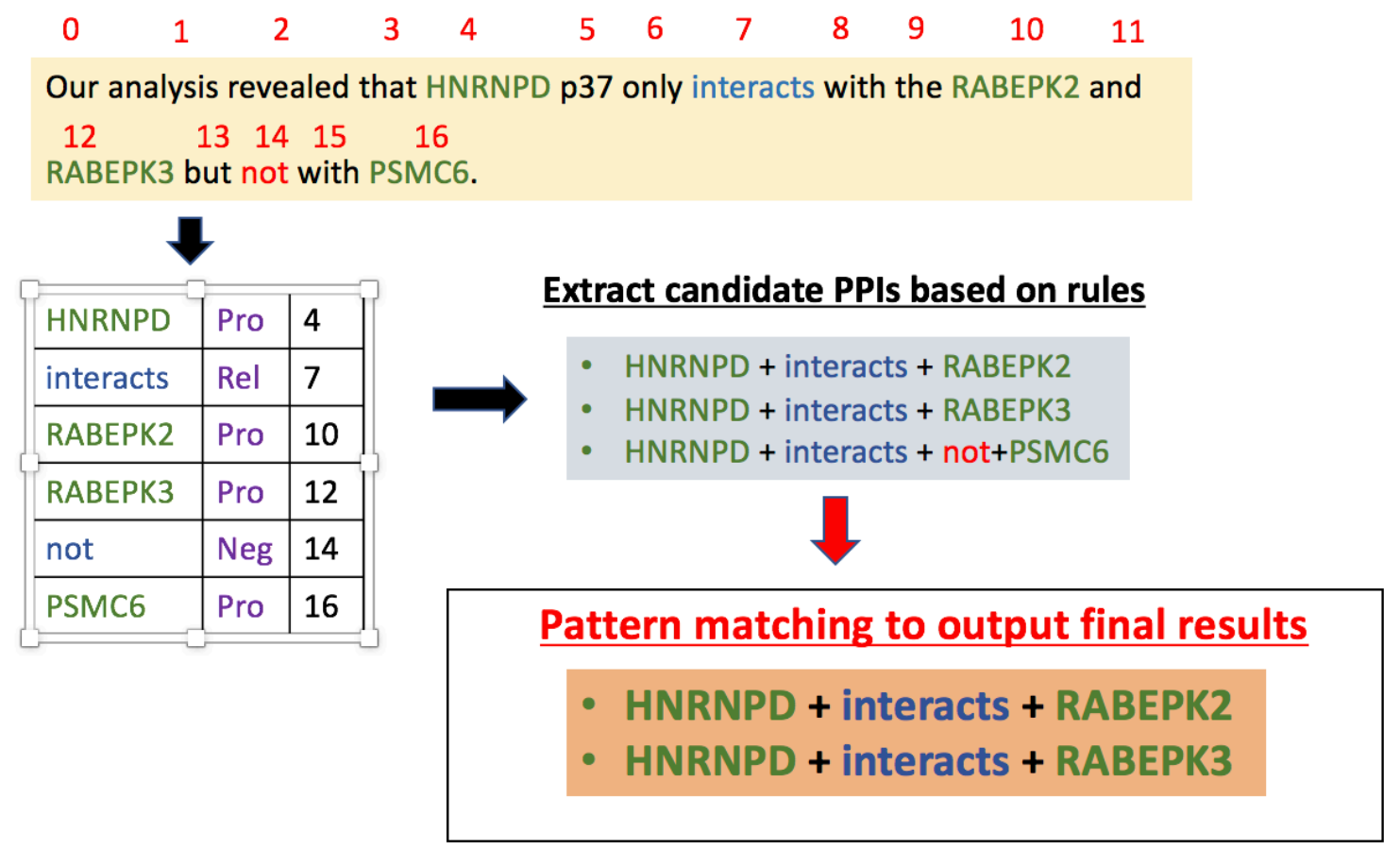

Figure 2. Algorithm to extract PPIs from complex sentences containing more than two protein entities [5]. First set of rules is applied on sentence to extract candidate PPIs. Pattern matching is done on candidate PPIs for final output.

\subsubsection{Machine-learning based methods}

To apply machine learning methods for extracting PPIs from biomedical literature, the task can be formulated as a binary classification problem where ML models are trained using annotated dataset (protein names, label for interaction) and a pair of protein names (an instance) from a new sentence can be predicted in terms of whether they interact using the model [15]. Over the last decade, a wide range of ML-based methods have been proposed to explore strategies of accomplishing task of PPIs extraction ranging from: 
- Using simple statistical inferences based on mere basic word embedding, such as bag-of-words (BoW);

- Feature-based and kernel-based ML models;

- Deep learning method, such as convolutional neural networks (CNN).

The first attempt made to extract PPIs using ML method in molecular biology was proposed by Kumlien et al. [30]. They used a Naïve Bayes model and a bag-of-words embedding method to represent sentences. One primary assumption by bag-of-words representation is the position of words in a sentence does not matter. Clearly this assumption does not hold in real biomedical text describing PPIs and this leads to an unsatisfactory performance.

As the advances in NLP, many sentence analysis tools and parsers become easily accessible and many ML-based methods have employed these tools to explore patterns of PPIs in a deeper level. Two main categories of methods are generally adopted to train machine learning models: the feature-based method [31-33] vs. kernel based method [34-36]. The common idea of these methods is to represent relation instances with either feature vectors (feature-based) or parsing trees or graphs (kernel-based). Then a discriminative classifier is used for a binary classification, for example, support vector machines (SVM) or conditional random fields (CRF) are choice of classifiers. Bui et. al [31] proposed a two-step ML-based method to extract PPIs. First, as mentioned in [4], based on relative position of relation word to two protein entities, they categorize sentences expressing two proteins interacting into different groups and designed rule-like procedures to extract candidate protein pairs from groups respectively. Next, they designed and extracted features from text, such as distance between two protein entities, constituency parsing height of two protein entities and POS 
tags of protein entities (some of features are shared over all five groups and some are specific for certain groups.) to train a SVM model which later is used to classify new instances.

Unlike feature-based methods where feature engineering is required, kernel-based methods utilize the structure of the instances represented as syntax trees or dependency graphs and apply kernel (similarity function) to quantify the similarity between two instances. Then a discriminative method is used to label new instances. The essence of kernel methods is the design of kernel function which can comprehensively represent the structure of instances and reflect the similarity between two instances [37]. Many kernel functions have been proposed exploring the best way to do this, such as shallow linguistic kernel [20], subtree kernel [38], all-paths graph kernel [34] and so on.

\subsubsection{An example}

Lately, deep learning methods have attracted much attention due to outstanding performance especially in fields of image processing and speech recognition. Researchers in biomedical text mining also started to explore how to apply deep learning models in the area. Convolutional neural networks (CNN) recently have achieved promising results in PPIs extraction task $[22,39,40]$. In this section, I illustrate as an example the method proposed by Peng et al. [22] which adopts multichannel dependency-based convolutional neural network model and achieved a F1 score of $63.5 \%$ and $65.3 \%$ in AIMed and BioInfer corpus. 


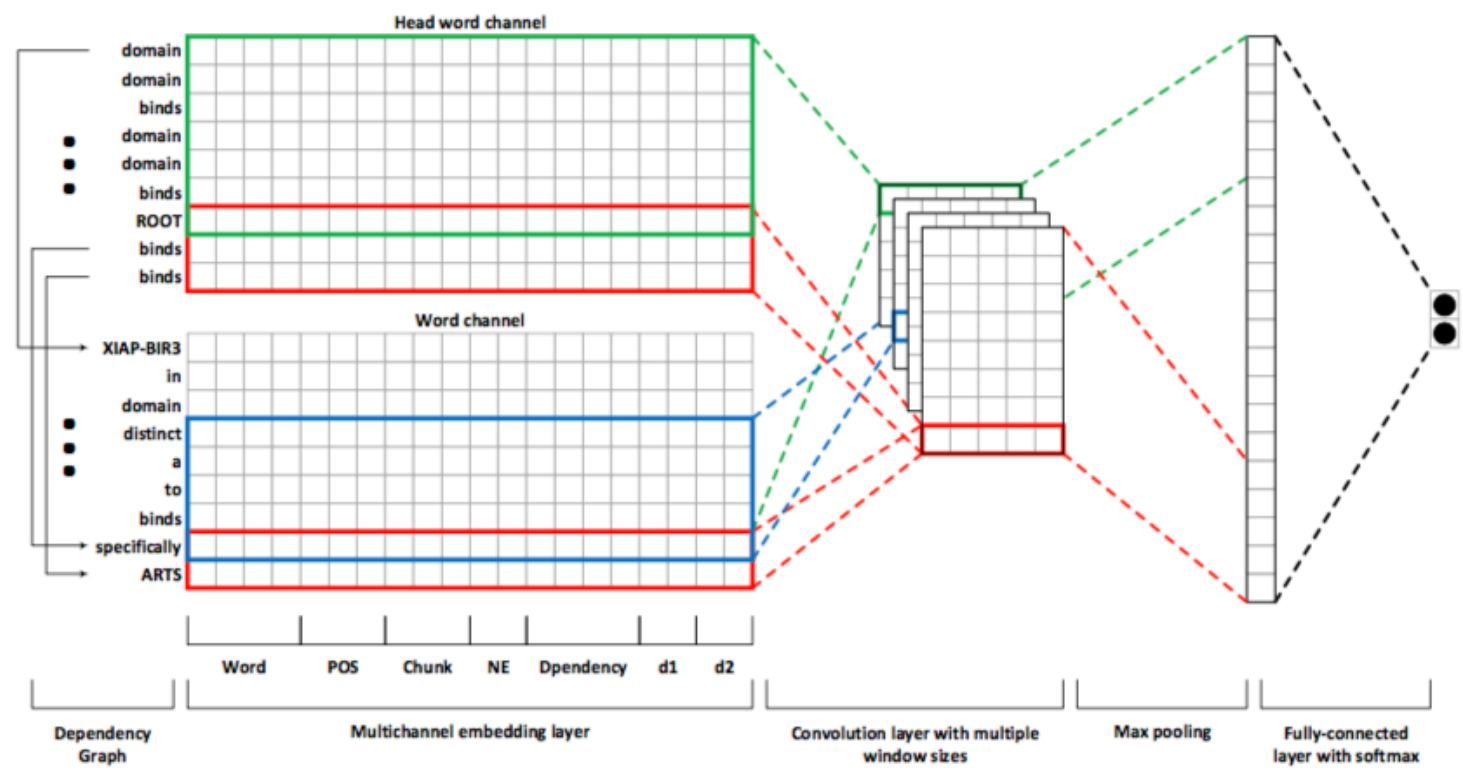

Figure 3. Overview of the CNN model proposed by [22]. Original text is transformed to feature channel and dependency channel which are then connected with multiple convolution layers. After max pooling, a fully- connected layer followed by a softmax is used to determine final classification. Reprinted from Deeping learning for extracting protein-protein interactions from biomedical literature. Proceedings of the 2017 Workshop on Biomedical Natural Language Processing

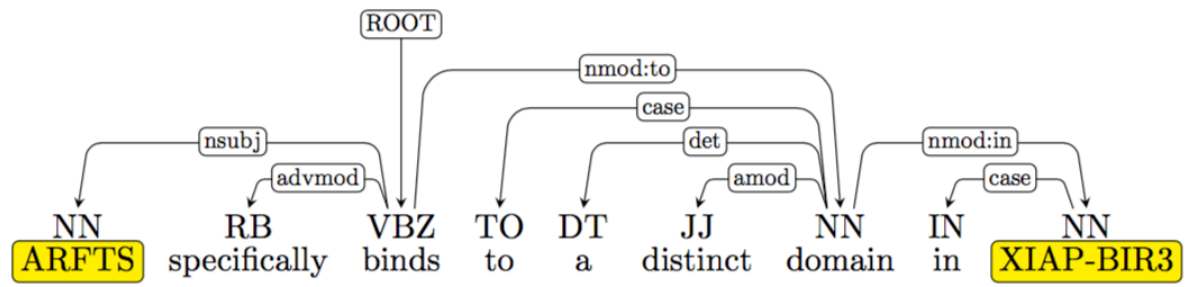

Figure 4. Dependency graph for the example sentence. The dependency parsing indicates the dependency relations between words in a sentence. Reprinted from Deeping learning for extracting protein-protein interactions from biomedical literature. Proceedings of the 2017 Workshop on Biomedical Natural Language Processing. [22]

Figure 3 illustrates the overview of CNN model proposed in [22]. Word embedding channel represents each word by concatenating its word embedding (pre-trained vectors learned on 
PubMed articles using word2vec [41]), POS, Chunk, dependency, position feature and named entity (indicate whether the word is the protein names of interest). Thus, the word embedding vector for each word is

$$
\mathrm{X}_{\mathrm{i}}=\mathrm{E}_{\text {word }}{ }^{\oplus \cdots \oplus \mathrm{E}_{\text {dependency }}}=[1,2 \ldots, \mathrm{n}]
$$

where $\mathrm{X}_{\mathrm{i}}$ denotes the $i$ th word in the sentence and $\oplus$ is concatenation operation. The innovation of [22] is the incorporation of 'head' word channel in embedding layer. For example, based on figure 4 ARFTS's head word is binds and specially's head word is also binds based on dependency graph of the sentence. This methodology intuitively conveys the dependency graph structure into CNN model. Also, together with convolution, this technique can better capture remote dependent relation compared to sliding window alone.

Then the convolution was applied to combine two channel. Let $x_{i: i+k-1}^{c}$ represent a window of size $\mathrm{k}$ in the sentence for channel $\mathrm{c}$. Then the output sequence of the convolution layer is

$$
\operatorname{con}_{i}=f\left(\sum w_{k}^{c} x_{i: i+k+1}^{c}+b_{k}\right)
$$

where $f$ is a rectify linear unit (ReLU) function and $b_{k}$ is the biased term. Both $w_{k}^{c}$ and $b_{k}$ are the parameters which will be learned during model training.

A fully connected layer (formula 4, represented as vector $O$ ) follows 1-max pooling (formula 3) for a whole coverage over extracted global features. The final softmax receives this vector $O$ as input and classify the PPIs with it.

$$
\begin{gathered}
m_{k}=\max _{1 \leq i \leq n-k+1}\left(\operatorname{con}_{i}\right) \\
O=w_{O}\left(m_{3}{ }^{\oplus m_{5}}{ }^{\oplus} m_{7}\right)+b_{O}
\end{gathered}
$$




\subsection{Extraction of PPIs Affected by Mutations}

Extraction of PPIs affected by mutations is a PPIs extraction task variant which was proposed by BioCreative VI in 2017 as a subtask of Precision Medicine Track [42]. As it is a new task, there are only limited attempts made to solve this problem.

[44] proposed by Tran et al. and [43] proposed by Chen et al. are the top two performing systems in BioCreative VI challenge and achieved a F1 score of around 3.7. [43] proposed a system adopting ML-based kernel method (SVM classifier, ASM graph kernel [45]) as previously discussed in chapter 2.2.3. [44] proposed a deep learning pipeline using CNN model to accomplish the task. Their method is similar to the example we discussed in chapter 2.2.3.1 but without 'head word' channel. Interestingly, in their proceeding report, they did not mention special detection for mutation words. 


\section{CHAPTER 3: METHOD}

In this chapter, I describe my proposed method to accomplish the task of extraction of PPIs affected by mutations. In this method, SVM machine learning model is trained using selected features extracted from training corpus. In the testing phase, two main steps are done to determine which protein pairs, if any, is the interacting pair and affected by mutations. In the first step, the classifier predicts if a new instance (a pair of protein) is interacting. Next, for the identified interacting protein pairs, mutation screening is applied to determine whether the pair is affected by mutations.

\subsection{System Overview}

To classify if a protein pair, which is extracted from a sentence, is interacting, a classifier is trained using training corpus; the protein names and interacting protein pairs are labeled in this corpus. The following steps are taken to train the classifier, as also depicted in (Figure 5):

- Extracting candidate PPI triplet, i.e. two protein entities and the interaction word connecting them, from sentences, which is described in detail in 3.3.

- Feature extraction for PPI triplet: Features used in this work are partially from [20, 49] and in combination with my proposed new sets of features as in the task of PPI candidate extraction, such as shortest path distance and negation using dependency parsing. Detailed description of features selected for model training is given in 3.4.

After we obtain our classifier, in the testing (classification) phase, we perform the following procedure to determine the pairs of proteins in each passage that are a) interacting and b) affected by mutations. First, each passage, i.e. abstracts of a biomedical 
literature, is separated into sentences and using an open-source protein recognition tool, which is similar to Name Entity Recognition (NER), the protein entities are identified and annotated as such (GNormPlus is used in the system which will be explained in 3.3). Then, as done in the training phase, the candidate protein pairs and the features are extracted and fed as input into the classifier to predict if the protein pair is interacting. Next, the positive instances from the classifier (that is, the interacting protein pairs) are further refined by 'mutation screening' step (tmVar open course is used to accomplish this task, described in 3.7). The outputs of the classifier are the protein pairs that are interacting and contain mutation related vocabulary.

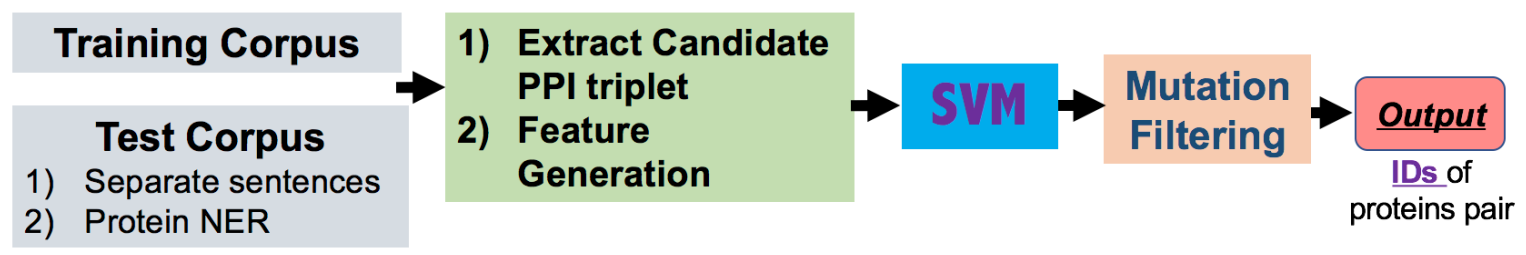

Figure 5. System overview. Trained SVM model is used to classify new instances. After protein NER, candidate PPI triplets are extracted from sentences and features for each triplet are generated. Only positive instances will be subject to mutation filtering for final output.

\subsection{Protein Name Recognition}

In any relation extraction task named entity recognition is always the first step to be accomplished. GNormPlus $[13]^{3}$ is a reliable open source tool for protein name recognition and acheves the best performance. Therefore, I leverage this tool for protein recognition from the text in this project. The output of this tool is both the annotated protein names and the Entrez ID of the protein.

\footnotetext{
${ }^{3}$ https://www.ncbi.nlm.nih.gov/research/bionlp/Tools/gnormplus/
} 


\subsection{Candidate Protein Pair Extraction}

I created a 'relation word' dictionary (seen in appendix1) containing words that convey the meaning of 'interacting'. The words in the dictionary contains 87 'root words' such as 'interact', 'bind', 'attach' and all their synonyms for each root word. The dictionary was created by combining relation keywords used by previous PPIs extraction works [49-51] and modifying based on the requirement of my work. As described in the related work chapter of this manuscript, there are three commonly defined abstract forms of Protein Protein Interaction (PPI) expression in the biomedical literature (was shown in Table 1, chapter 2). Based on these three abstract forms, all possible candidate PPI triplets are extracted from sentences described as following: After parsing the sentence with Stanford Parser, all nouns and verbs that have a match in the 'relation word' dictionary are identified, along with the location in the document. The locations of protein entities are given in training corpus. With these information, PPI triplet (two protein entities and the relation word which indicates 'interaction') can be extracted from sentences. After extracting PPI triplet, a set of features for each of PPI triplet is generated, such as distance features, and dependency features. These features are used for classifier training; detail is provided in 3.4. For complex sentences where there are more than two protein entities in the sentence, every possible set of PPI triplet will be extracted. For example, in a sentence like $\mathrm{P}_{1}$-tokens- $\mathrm{P}_{2}$-tokens-REL(I)- $\mathrm{P}_{3}$, three sets of PPI triplets will be formed as following: $\left\{\mathrm{P}_{1} \mathrm{P}_{2} \mathrm{I}, \mathrm{P}_{1} \mathrm{IP}_{3}, \mathrm{P}_{2} \mathrm{IP} \mathrm{P}_{3}\right\}$ 


\subsection{Features}

In this section, we describe features used for classification. The feature sets are generated along with extracting PPI triplets from sentences. In the model training phase, these features and the labels which indicate if the protein pair is interacting, are used to train the model to distinguish interacting protein pairs from non-interacting pairs. In the classification/test phase, the same set of features are extracted and fed into the trained classifier.

Relation word - the relative position of relation word to two protein entities (if the relation word is in the middle of two protein entities or is located in the first/last position) is an important feature for determining the PPI triplet.

Protein entity frequency - the number of protein entities in the sentence is also a feature. Intuitively, sentences describing protein-protein interaction may contain a certain number of protein entities.

Distance - three distance features: 1) distance between two protein entities; 2) distance of first protein entity to relation word; 3) distance of second protein entity to relation word. For example, in the figure 6, for $P R O 1$ and $P R O 2$, these three features are 1, 1 and 3. Height- For each triplet, we count each protein entities constituency height. As shown in Figure 6, the height of a protein entity refers to the number of nodes between itself to root node. 


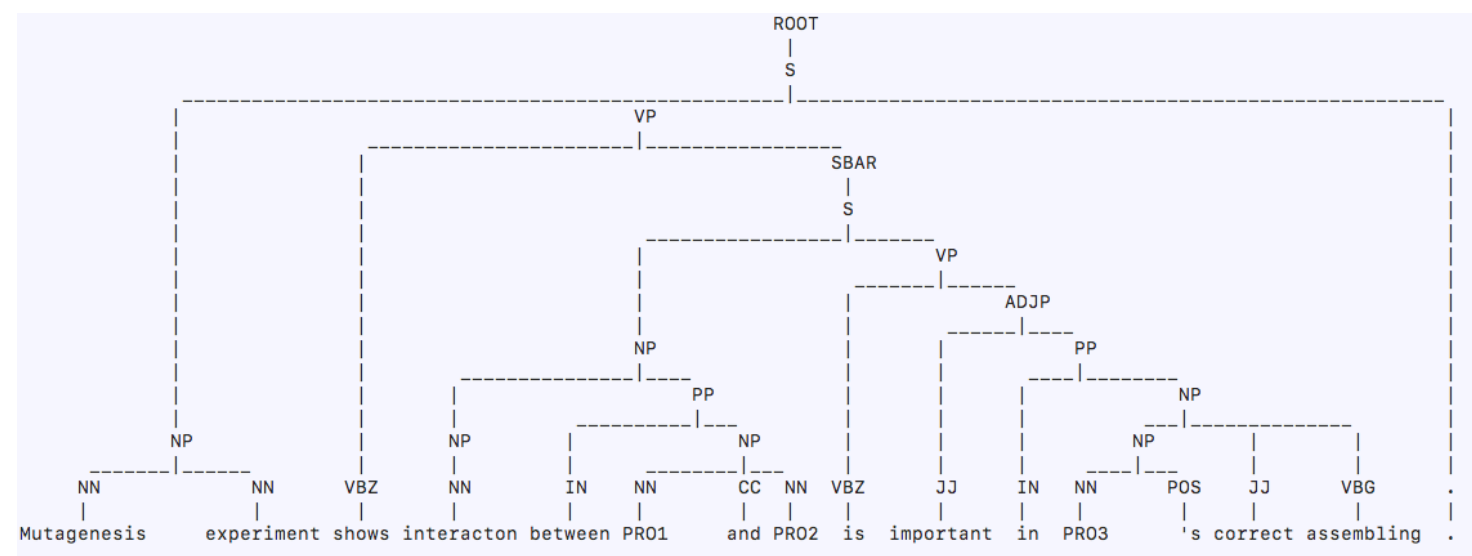

Figure 6. The Stanford parser output. The constituency parsing annotates each syntactic role for each phrase in a sentence.

Dependency Distance- also called shortest path distance. we count the minimum number of edges connecting two protein entities in dependency parsing graph as two protein entities' dependency distance. For example, in Figure 7, the dependency distance for PRO1 and $\mathrm{PRO} 2$ is 2. Earlier published research has not used this feature in the task PPIs extraction. Negation- For the relation words, if there is a 'neg' edge, then it is detected as negation. As shown in Figure 7, there is cannot in front of relation word interact which indicates a 'neg' edge going out from the relation word. Earlier published research has not used this feature in the task of PPIs extraction.

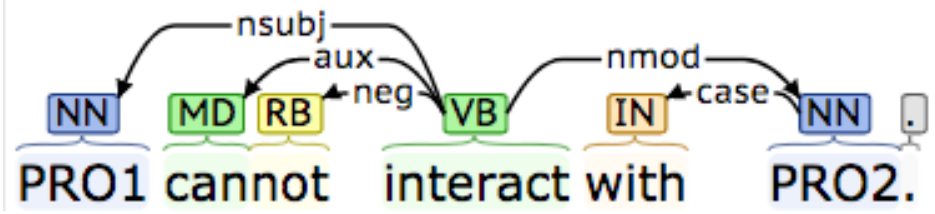

Figure 7. Dependency graph for the example sentence. The dependency distance PRO1 and PRO2 is 2 and 'interact' is negated because there is a negative edge leaving from it. 


\subsection{Machine Learning Model}

In this work, we use SVM with a default radial basis function (RBF) kernel as machine learning model for classification task. SVM is a supervised ML algorithm. With annotated training data, the model learns a linear decision boundary which will be used to classify new instances. In order to deal with non-linear data, kernel functions are designed to transform non-linear data into linear and RBF is one of widely used kernel functions. Features mentioned above (3.2) are combined into a single feature vector for training the classification model. The classifier learns whether a protein pair is interacting or not given features generated for each PPI triplet and the labels which indicate if the protein pair is interacting or not.

\subsection{Mutation Screening}

The positive instances which are determined by the classifier as PPI are subject to the mutation screening to determine if the interacting protein pair is affected by mutations. We use an open source tool, namely tmVar [16] to determine whether there is a mutation mention in the sentence. In biomedical articles mutations can be expressed in the following ways, which tmVar can capture:

- T467M: it indicates the amino acid at position 467 changed from $\mathrm{T}$ to M. Here $T$ and $M$ represents the respective type of amino acid. There are two sets of abbreviations for a total of 20 type of amino acids. This example represents one letter method. The other method uses three letters to represent one type of amino acid and the example is as the following:

- Arg505Lys: amino acid at position 505 changed from Arg to Lys. 
Furthermore, any word rendering the meaning of mutation is also considered as a mutation mention, such as mutant, mutating, mutagenesis, etc. 


\section{CHAPTER 4: EXPERIMENTATION RESULTS AND ANALYSES}

In this chapter, I evaluate my proposed method using the BioCreative VI Precision Medicine Track testing dataset. The chapter starts with providing a description of the benchmark dataset and experimental setup. The evaluation metrics are described in 4.3. Finally, the results and analysis are given in 4.4 .

\subsection{Dataset}

BioCreative VI PM Track Training: As shown in Table 2, this corpus consists of 597 abstracts of biomedical articles describing research results of PPIs. There are 752 pairs of PPIs which are affected by mutations in 597 passages. These pairs are labeled; an example is shown in Figure 8, where 881908 and 881982 are the Entrez IDs of the interacting protein pair affected by mutations. In the text, all occurrences of this pair of protein names are also annotated as marked in purple. Other protein mentions are not annotated. When using this corpus for model training, we started with using GNormPlus to annotate other protein names in the text. Only originally labeled protein names occurring in the same sentences are seen as positive instance (interacting protein pairs). Others are all negative instances.

Table 2. Statistics of BioCreativeVI PM Track training and testing corpus.

\begin{tabular}{|c|c|c|c|}
\hline Dataset & \# of Articles & \# of relations & Format \\
\hline Training & 597 & 752 & Xml/json \\
\hline Testing & 688 & 930 & Xml/json \\
\hline
\end{tabular}


TITLE:

Cochaperone interactions in export of the type III needle component PscF of Pseudomonas aeruginosa.

ABSTRACT:

Type III secretion (T3S) systems allow the export and translocation of bacterial effectors into the host cell cytoplasm. Secretion is accomplished by an 80-nm-long needle-like structure composed, in Pseudomonas aeruginosa, of the polymerized form of a 7-kDa protein, PscF. Two proteins, $\mathrm{PscG}$ and $\mathrm{PscE}$, stabilize PscF within the bacterial cell before its export and polymerization. In this work we screened the 1,320-A(2) interface between the two chaperones, PscE and PscG, by site-directed mutagenesis and determined hot spot regions that are important for T3S function in vivo and complex formation in vitro. Three amino acids in PscE and five amino acids in PscG, found to be relevant for complex formation, map to the central part of the interacting surface. Stability assays on selected mutants performed both in vitro on purified PscE-PscG complexes and in vivo on P. aeruginosa revealed that PscE is a cochaperone that is essential for the stability of the main chaperone, PscG. Notably, when overexpressed from a bicistronic construct, $\mathrm{PscG}$ and PscF compensate for the absence of PscE in cytotoxic P. aeruginosa. These results show that all of the information needed for needle protein stabilization and folding, its presentation to the $\mathrm{T} 3$ secreton, and its export is present within the sequence of the PscG chaperone.

Relation name Relation type Bio-entities

PPIm Gene_Gene 881908(PscE) | 881982(PscG) Report an issue

Figure 8. A snapshot of visualization of BioCreative VI PM Track Training Corpus

[46]. The interacting protein pair affected by a mutation is annotated at the document level.

AIMed+BioInfer: These two corpora are sentence-based and were created for training and testing PPI extraction $[47,48]$. As shown in Figure 9, for each sentence, protein names and whether these proteins are interacting are annotated. Statistics of these two corpora are shown in Table. 3.

Table 3. Statistics of AIMed and BioInfer Corpora

\begin{tabular}{|l|l|l|l|}
\hline Corpus & \#Sentences & \# Sentences with & \# of PPIs \\
& & PPIs & \\
\hline AIMed & 1955 & 887 & 1000 \\
\hline BioInfer & 1100 & 1100 & 1434 \\
\hline
\end{tabular}




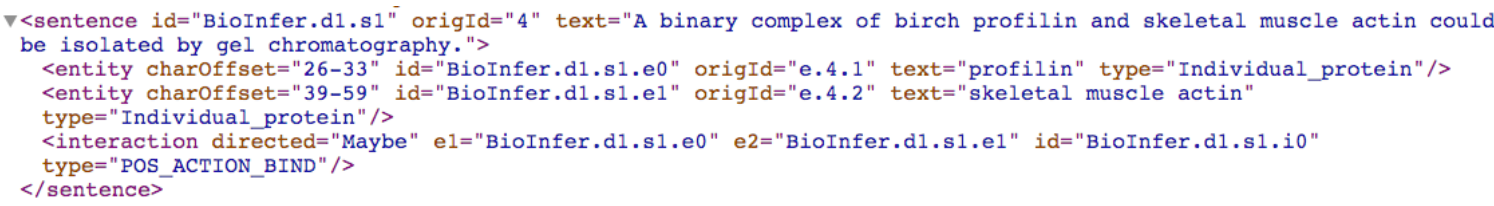

Figure 9. A snapshot of converted BioInfer corpus. All protein names and interacting protein pairs are annotated.

BioCreative VI PM Track Testing, as shown in Table 2, there are 688 abstracts of PubMed biomedical articles containing 930 PPIs affected by mutations. For each positive relation, the ground truth of Entrez IDs of two proteins are provided.

\subsection{Experimental Setup}

As explained in Chapter 2, I used GNormaPlus[13] for protein name recognition and Entrez ID conversion; to obtain constituency parsing and part-of-speech, we used Stanford coreNLP package [52]. For dependency parsing, we used spacy and networkx Python packages. All the results are based on SVM sklearn implementation with a default BRF kernel setting (note that two other classifier XGBoost and MLP were evaluated and due to the better performance of SVM it was determined to use SVM). We used tmVar[16] for mutation recognition.

\subsection{Evaluation Metrics}

The commonly used evaluation metrics precision, recall and F-score are used in this study to evaluate the performance PPI detection and mutation detection. Let TP denote numbers of true positives, FP denote the number of false positives and FN denote the number of false negatives. The measures are defined as follows:

Recall $=\mathrm{TP} /(\mathrm{TP}+\mathrm{FN})$ 
Precision $=\mathrm{TP} /(\mathrm{TP}+\mathrm{FP})$

$\mathrm{F}$-score $=2 *$ Recall $*$ Precision $/($ Recall + Precision $)$

\subsection{Result and Analysis}

\subsubsection{Comparison with existing systems}

We show evaluation results for all six participating teams in the BioCreative VI PM track and baseline method proposed by the organizer in Table 4. The baseline method (row 7) which is a co-occurrence-based approach, considers the existence of any two entities in one sentence as an indication of relation and achieves a $\mathrm{F} 1$ of 0.23 . The best performing system (Row 1) utilizes the convolutional neural network (CNN) model with the word2vec word embedding method (F1 0.37). Another comparable system with respect to F1 measure (Row 2) is a system based on approximate subgraph matching kernel, achieving F1 of 0.37 but with a trade-off in precision and recall when compared with the best performing system of Row 1 . The reason why they achieved better performance should be that $\mathrm{CNN}$ model and the kernel method can do a more comprehensive capturing for characteristics of PPIs. Therefore, the trained classifier is able to achieve a high recall rate without losing the rate of precision. However, one drawback of these automatic feature generation methods is that they lack interpretability compared to feature rich methods. Rows 3, 4, 6 also attempted to explore variants of neural network to accomplish the task, however their models did not perform well. Row 5 is a feature-based ML-method using XGBOOST which suffers from low performance. Among the existing 7 systems, including the baseline, the performance of my system (Row 8), although not reaching the performance of the top system, can be placed below the two best performing state-of-the-art systems (F1: 0.33 as compared to 0.37 ; R: 
0.34 as compared to 0.33 ; and P:0.32 as compared to 0.45 ). It also achieved almost 50\% improvement in F-score compared to the baseline method.

Table 4. Evaluation results for my method and participating teams in BioCreative VI PM Track [42].

\begin{tabular}{|c|c|c|c|}
\hline Method & Precision & Recall & F1 \\
\hline 1. Convolutional Neural Network & 0.4544 & 0.3161 & 0.3729 \\
\hline 2. Approximate Subgraph Matching Kernel & 0.4252 & 0.3301 & 0.3717 \\
\hline 3. Representation Learning +RNN & 0.236 & 0.1989 & 0.2159 \\
\hline 4. Entity-enhanced Hierarachical Attention & 0.1135 & 0.5387 & 0.1875 \\
Neural Networks (EHANN) & & & \\
\hline 5. Feature-based: XGBOOST & 0.0803 & 0.2742 & 0.1242 \\
\hline 6. Long Short-term Memory & 0.1061 & 0.0376 & 0.0556 \\
\hline 7. baseline & 0.146 & 0.5215 & 0.2282 \\
\hline 8. This work & 0.3198 & 0.3398 & 0.3296 \\
\hline
\end{tabular}

\subsubsection{Effect of auxiliary dataset}

The SVM model was trained using BioCreative VI PM training corpus and AIMed+BioInfer (same features extracted for training) and evaluated respectively. The result shown in Table 5 indicates that combined auxiliary corpora help to achieve better performance for the task. The BioCreative corpus only provides annotations for the proteins that are affected by mutations; other protein entities that are not annotated are 
labeled using protein recognition tools. In my experimentations, I used GNormPlus which is the best protein annotating tool currently available for protein recognition. I evaluated the performance of GNormPlus in BioCreative dataset and observed an $81.2 \%$ recall rate in the training dataset, and hence it failed to capture around $20 \%$ of protein entities. Also, in a sentence, even though two protein entities are annotated, the context may not express that they are interacting. For example, in Figure 7, in the sentence "two proteins, PscG and PscE, stabilize PscF within the bacterial cell before its export and polymerization, ” PscG and PscE are annotated as protein entities although the sentence does not convey that they are interacting. However, AIMed and BioInfer datasets provide annotations for all proteins in each sentence and also label each interacting pair. This additional labeling information from these two datasets enables the combined auxiliary corpora to improve the classifier's training by better capturing the characteristics of true PPIs as well as filtering false positives, thus achieving a better system performance compared to the BioCreative training corpus.

Table 5. Comparison of the effectiveness of system trained by different training corpus.

\begin{tabular}{|l|l|l|l|l|}
\hline Method & Corpus & Precision & Recall & F1 \\
\hline SVM & BioCreative VI & 0.3396 & 0.2398 & 0.2997 \\
& Training & & & \\
\hline SVM & AIMed+BioInfer & 0.3198 & 0.3398 & 0.3296 \\
\hline
\end{tabular}




\subsubsection{Effect of negation detection feature and shortest path feature}

Finally, I tested the effect of two features introduced in my work, the negation detection feature and the dependency distance feature. As seen in Table 6. using auxiliary corpora, adding these two features individually or simultaneously to the originally selected feature set both lead to improvements to the system. In the last row, we can see two new features help to improve F1 score by $12 \%$. This result underscores the effectiveness of these two features in classifier training and overall system performance.

Table 6. Comparison of the effectiveness of added features used for classifier training.

\begin{tabular}{|c|c|c|c|}
\hline Features & Precision & Recall & F1 \\
\hline selected features from [20,49] & 0.2798 & 0.3096 & 0.2940 \\
\hline selected features from [20,49] +negation & 0.3000 & 0.3096 & 0.3050 \\
\hline $\begin{array}{c}\text { selected features from [20,49] } \\
+ \text { dependency }\end{array}$ & 0.3001 & 0.3204 & 0.3100 \\
\hline $\begin{array}{c}\text { selected features from } \\
{[20,49]+\text { negation+dependency }}\end{array}$ & 0.3198 & 0.3398 & 0.3296 \\
\hline
\end{tabular}

\subsection{Error Analysis on False Positives and False Negatives}

The following reasons have been found to contribute to a good portion of errors:

- As mentioned above, GNormPlus's recall rate in the testing dataset is $73.4 \%$. It means almost $30 \%$ of protein names are not annotated nor given the correct id by the tool. 
- In ground truth of the testing data, we found that 84 out of 930 relations consist of only one protein ID. After tracking back to the original articles, we found that these sentences talk about protein self-interaction (subunit of a protein molecule is interacting with the other subunit of the protein) and this situation cannot be captured by my extraction system.

- The system is prone to give some false positive predictions when there are more than two protein entities in the sentence, for example:

- $\mathrm{mDia}_{\mathrm{PRO} 1}$ binds profilin ${ }_{\mathrm{PRO}}$ likely to promote actin $_{\mathrm{PRO}}$ polymerization. My system can predict mDia and profilin to be interacting correctly. However, it will also give profilin and actin as positive which is a false positive.

- Under some special cases, the system may miss some true positive instances . For example, "TEXG56 cannot bind to P53 due to C786Y mutation" should be predicted as positive. However, the classifier prediction for TEXG56 (PRO1) and P53 (PRO2) is negative because there is a negation word in front of the relation word bind. Thus, this protein pair cannot be classified as a positive relation in the system.

- Correct outputs of the system should be pairs of interacting proteins for which there is a mutation within either protein that affects the interaction. After a close observation of false positive predictions, a situation is identified to be difficult for the system to predict correctly. As seen in the following example:

- In three of the cell lines with CTNNB1 mutations, complexes of betacatenin $_{\text {PRO1 }}$ with alpha-catenin ${ }_{\text {PRO2 }}$ were detectable.

beta-catenin and alpha-catenin are predicted as positive by the system, however, this is a false positive. It is true that beta-catenin and alpha-catenin are interacting based 
on the meaning of the sentence and there is a mutation mentioning in the sentence. However, if read very carefully, I found the 'CTNNB1' mutation is not a mutation either in catenin or alpha-catenin. Therefore, this pair should not be output as a positive answer by the system.

In future work, I would like to develop methods to solve these problems and improve system performance.

\subsection{Summary of Results}

In summary, the experiments demonstrate that my approach obtained good performance in the Biocreative VI dataset. After evaluating two training corpora, evaluation results show that an auxiliary corpus helps to achieve better system performance. Additional features such as dependency also improved the results. 


\section{CHAPTER 5: CONCLUSION}

In this thesis, I proposed a feature-rich supervised method to accomplish the task of extracting Protein-Protein-Interactions (PPIs) affected by mutations from biomedical literature. First, a model was trained with a wide range of features (part-of-speech, dependency, semantic, negation etc.) and used to predict if a pair of proteins is interacting in new instances. Next, a 'mutation screening' step was incorporated as a filter to determine the outcome. Experiments on BioCreative VI Precision Medicine (PM) track dataset demonstrated the effectiveness of the proposed method in accomplishing the task.

Features for model training were carefully selected from the state-of-the-art PPI extraction work and new features were incorporated into the system. These selected features have shown a good coverage to represent characteristics of expression for PPI in biomedical literatures. The evaluation results showed that selected features help to achieve a competitive performance. Additionally, new features, i.e. negation terms and dependency distance, were tested individually and in combination. Their effectiveness for performance enhancement was proved by the evaluation result. This 'mutation screening' step filtered out significant amount of false positives, thus achieving a higher overall F-score.

In addition, BioCreative VI training corpus and combined AIMed+BioInfer auxiliary corpus were used for classifier training. Evaluation results indicate that classifier trained using auxiliary corpus achieves a better system performance. AIMed and BioInfer were both created for PPI extraction system development and testing. Their advantage over BioCreative VI can be summarized as the following: i) All protein entities are annotated; 
ii) the two interacting protein entities are labeled. These two advantages enable this combined auxiliary corpus to train a better classifier for this research task.

The NLP tools and machine learning packages used in this work are publicly available and thus it is rather easy to replicate this work. Furthermore, the proposed features can also be applied to other biomedical relation extraction tasks, such as ligand-receptor extraction, gene-disease association, etc.

In summary, the contributions of this thesis are as follows:

- Proposed a feature-rich supervised model to accomplish the task of extraction of PPIs affected by mutations. Mutation detection phase is incorporated as a filter to determine PPIs affected by mutations.

- Proposed and evaluated the effectiveness of utilizing an auxiliary dataset (AIMed+BioInfer) for training the classifier for the task, showing a better performance due to the improved labeling of PPIs and mutations.

- Favorably evaluated the combination of existing features and the additional negation and distance features for model training in extracting PPIs from biomedical literature.

In the future, I would like to explore more features suitable for the model and further improve the system performance. Furthermore, I am interested in exploring methods using deep learning in combination with the features used in this work. Finally, I am interested to 
apply and adopt my methods to other biomedical relation tasks such as the drug-protein interaction task. 


\section{APPENDIX: RELATION WORD DICTIONARY}

\begin{tabular}{|c|c|}
\hline Root Word & Derivations (Relation Keywords) \\
\hline Abolish & abolish, abolishes, abolished, abolishing \\
\hline Accelerate & accelerate, accelerates, accelerated, accelerating \\
\hline Acceptor & acceptor \\
\hline Accumulate & $\begin{array}{l}\text { accumulate, accumulates, accumulated, accumulating, } \\
\text { accumulation }\end{array}$ \\
\hline Acetylate & acetylate, acetylates, acetylated, acetylating, acetylation \\
\hline Activate & activate, activates, activiated, activating, activation, activator \\
\hline Affect & affect, affects, affected, affecting \\
\hline Alter & alter, alters, altered, altering, alteration \\
\hline Amplify & amplify, amplifies, amplified, amplifying, amplification \\
\hline Apoptosis & apoptosis \\
\hline Assemble & assemble, assembles, assembled, assembling \\
\hline Associate & associate, associates, associated, associating, association \\
\hline Attach & attach, attaches, attached, attaching, attachment \\
\hline Attack & attack, attacks, attacked, attacking \\
\hline Bind & bind, binds, bound, binding \\
\hline Block & block, blocks, blocked, blocking \\
\hline Carbamoylate & $\begin{array}{l}\text { carbamoylate, carbamoylates, carbamoylated, carbamoylating, } \\
\text { carbamoylation }\end{array}$ \\
\hline Carboxylate & $\begin{array}{l}\text { carboxylate, carboxylates, carboxylated, carboxylating, } \\
\text { carboxylation }\end{array}$ \\
\hline Catalyze & catalyze, catalyzes, catalyzed, catalyzing \\
\hline Cleave & cleave, cleaves, cleaved, cleaving \\
\hline $\begin{array}{l}\text { Co- } \\
\text { immunoprecipitate }\end{array}$ & $\begin{array}{l}\text { co-immunoprecipitate, co-immunoprecipitates, co- } \\
\text { immunoprecipitated, co-immunoprecipitating, co- } \\
\text { immunoprecipitation, co-immunoprecipitations }\end{array}$ \\
\hline Complex & complex, complexes, complexed, complexing, complexation \\
\hline Conjugate & conjugate, conjugates, conjugated, conjugating, conjugation \\
\hline Contact & contact, contacts, contacted, contacting \\
\hline Deaccetylate & $\begin{array}{l}\text { deaccetylate, deaccetyltes, deaccetylated, deaccetylating, } \\
\text { deaccetylation }\end{array}$ \\
\hline Deaminate & deaminate, deaminates, deaminated, deaminating, deamination \\
\hline Decarboxylate & $\begin{array}{l}\text { decarboxylate, decarboxylates, decarboxylated, decarboxylating, } \\
\text { decarboxylation }\end{array}$ \\
\hline Decrease & decrease, decreases, decreased, decreasing \\
\hline Dehydrate & dehydrate, dehydrates, dehydrated, dehydrating, dehydration \\
\hline Dehydrogenate & $\begin{array}{l}\text { dehydrogenate, dehydrogenates, dehydrogenated, dehydrogenating, } \\
\text { dehydrogenation }\end{array}$ \\
\hline Demethylate & $\begin{array}{l}\text { demethylate, demethylates, demethylated, demethylating, } \\
\text { demethylation }\end{array}$ \\
\hline Dephosphorylate & $\begin{array}{l}\text { dephosphorylate, dephosphorylates, dephosphorylated, } \\
\text { dephosphorylating, dephosphorylation }\end{array}$ \\
\hline
\end{tabular}




\begin{tabular}{|c|c|}
\hline Deplete & deplete, depletes, depleted, depleting, depletion \\
\hline Disassemble & disassemble, disassembles, disassembled, disassembling \\
\hline Discharge & discharge, discharges, discharged, discharging \\
\hline Dock & dock, docks, docked, docking \\
\hline Down-regulate & $\begin{array}{l}\text { down-regulate, down-regulates, down-regulated, down-regulating, } \\
\text { down-regulation }\end{array}$ \\
\hline Downregulate & $\begin{array}{l}\text { downregulate, downregulates, downregulated, downregulating, } \\
\text { downregulation }\end{array}$ \\
\hline Elevate & elevate, elevates, elevated, elevating, elevation \\
\hline Enhance & enhance, enhances, enhanced, enhancing \\
\hline Express & express, expresses, expressed, expressing, expression \\
\hline Formylate & formylate, formylates, formylated, formylating, formylation \\
\hline Glycosylate & $\begin{array}{l}\text { glycosylate, glycosylates, glycosylated, glycosylating, } \\
\text { glycosylation }\end{array}$ \\
\hline Hasten & hasten, hastenes, hastening, hastened \\
\hline Heterodimerize & $\begin{array}{l}\text { heterodimerize, heterodimerizes, heterodimerizing, } \\
\text { heterodimerized, heterodimerization, heterodimer, heterodimers }\end{array}$ \\
\hline Homodimerize & $\begin{array}{l}\text { homodimerize, homodimerizes, homodimerizing, homodimerized, } \\
\text { homodimerization, homodimer, homodimers }\end{array}$ \\
\hline hydrolyse & hydrolyse, hydrolyses, hydrolysing, hydrolysed, hydrolysis \\
\hline Inactivate & inactivate, inactivates, inactivated, inactivating, inactivation \\
\hline Incite & incite, incites, incited, inciting \\
\hline Induce & induce, induces, induced, inducing, induction \\
\hline Infect & infect, infects, infected, infecting \\
\hline Influence & influence, influences, influencing, influenced \\
\hline Inhibit & inhibit, inhibits, inhibited, inhibiting, inhibition, inhibitors \\
\hline Initiate & initiate, initiates, initiated, initiating, initiation \\
\hline Interact & interact, interacts, interacts, interacting, interaction \\
\hline Impair & impair, impairs, impaired, impairing \\
\hline Isomerize & isomerize, isomerizes, isomerized, isomerizing, isomerization \\
\hline Ligate & ligate, ligates, ligated, ligating, ligation, ligand \\
\hline Mediate & mediate, mediates, mediated, mediating \\
\hline Methylate & methylate, methylates, methylated, methylating, methylation \\
\hline Modify & modify, modifies, modified, modifying, modification \\
\hline Modulate & modulate, modulates, modulating, modulated \\
\hline Myogenesis & myogenesis \\
\hline Overexpress & $\begin{array}{l}\text { overexpress, overexpresses, overexpressed, overexpressing, } \\
\text { overexpression }\end{array}$ \\
\hline Oxidize & oxidize, oxidizes, oxidized, oxidizing, oxidation \\
\hline Pair & pair, pairs, paired, paring \\
\hline Participate & participate, participates, participated, participating, participation \\
\hline Peroxidize & peroxidize, peroxidizes, peroxidized, peroxidizing, peroxidation \\
\hline Phosphorylate & $\begin{array}{l}\text { phosphorylate, phosphorylates, phosphorylated, phosphorylating, } \\
\text { phosphorylation }\end{array}$ \\
\hline Prevent & prevent, prevents, prevented, preventing \\
\hline
\end{tabular}




\begin{tabular}{|l|l|}
\hline Produce & produce, produces, produced, producing, production \\
\hline Promote & promote, promotes, promoted, promoting, promotion \\
\hline React & react, reacts, reacted, reacting, reaction \\
\hline Recognize & recognize, recognizes, recognized, recognizing, recognition \\
\hline Recruit & recruit, recruits, recruited, recruiting \\
\hline Regulate & regulate, regulates, regulated, regulating, regulation \\
\hline Replace & replace, replaces, replaced, replacing \\
\hline Repress & repress, represses, repressed, repressing, repression \\
\hline Severe & severe, severes, severed, severing \\
\hline Stimulate & $\begin{array}{l}\text { stimulate, stimulates, stimulated, stimulating, stimulation, } \\
\text { stimulator }\end{array}$ \\
\hline Substitute & substitute, substitutes, substituted, substituting, substitution \\
\hline Suppress & suppress, suppresses, suppressed, suppressing, suppression \\
\hline Tether & Tether, tethers, tethered, tethering \\
\hline Transactivate & $\begin{array}{l}\text { transactivate, transactivates, transactivated, transactivating, } \\
\text { transactivation, transactivator }\end{array}$ \\
\hline Transaminate & $\begin{array}{l}\text { transaminate, transaminates, transaminated, transaminating, } \\
\text { transamination }\end{array}$ \\
\hline Ubiquitinate & $\begin{array}{l}\text { ubiquitinate, ubiquitinates, ubiquitinated, ubiquitinating, } \\
\text { ubiquitination }\end{array}$ \\
\hline Upregulate & $\begin{array}{l}\text { upregulate, upregulates, upregulated, upregulating, upregulation, } \\
\text { upregulator }\end{array}$ \\
\hline Up-regulate & $\begin{array}{l}\text { up-regulate, up-regulates, up-regulated, up-regulating, up- } \\
\text { regulation, up-regulator }\end{array}$ \\
\hline
\end{tabular}




\section{REFERENCES}

[1] Adam A. Friedman, Anthony Letai, David E. Fisher, Keith T. Flaherty (2015) Precision medicine for cancer with next-generation functional diagnostics, Nature Review Cancer $15,747-756$

[2] Suresh H. Moolgavkar, Alfred G. Knudson (1981) Mutation and Cancer: A Model for Human Carcinogenesis, JNCI: Journal of the National Cancer Institute, Volume 66, Issue 6, Pages 1037-1052

[3] Hoffmann R, Krallinger M, Andres E, Tamames J, Blaschke C, et al. (2005) Text mining for metabolic pathways, signaling cascades, and protein networks. Sci STKE 2005: pe21.

[4] Tikk D, Thomas P, Palaga P, Hakenberg J, Leser U (2010) A Comprehensive Benchmark of Kernel Methods to Extract Protein-Protein Interactions from Literature. PLoS Comput Biol 6(7): e1000837. doi:10.1371/journal.pcbi.1000837

[5] Kalpana Raja, Suresh Subramani, and Jeyakumar Natarajan. (2013) PPInterFinder-a mining tool for extracting causal relations on human proteins from literature. Database (2013) Vol. 2013: article ID bas052; doi:10.1093/database/bas052.

[6] Bunescu,R. and Mooney,R. (2005) Subsequence kernels for relation extraction. In Proceedings of the 19th conference on Neural Information Processing Systems. Vancouver, Canada.

[7] Miwa,M. et al. (2009) Protein-protein interaction extraction by leveraging multiple kernels and parsers. Int. J. Med. Inform., 78, e39-e46.

[8] Deyu Zhou *, Yulan He (2008) Extracting interactions between proteins from the 
literature Journal of Biomedical Informatics 41 393-407

[9] PubMed-overview. http://www.ncbi.nlm.nih.gov/entrez/query/static/ overview.html.

[10] Tomohiro Mitsumori, Sevrani Fation, Masaki Murate, Kouichi Doi and Hirohumi Doi (2005)Gene/protein name recognition based on support vector machine using dictionary as features. BMC Bioinformatics 6(Suppl 1):S8 doi:10.1186/1471-2105-6-S1-S8

[11] Fukuda K, Tamura A, Tsunoda T, Takagi T (1998) Toward Information Extraction: Identifying protein names from biological papers. Proceedings of the Pacific Symposium on Biocomputing:707-718.

[12] Franzén K, Eriksson G, Asker FOL, Lidén P, Cöster J (2002) Protein names and how to find them. International Journal of Medical Informatics 67:49-61.

[13] Chih-Hsuan Wei, Hung-Yu Kao, and Zhiyong Lu. (2015) GNormPlus: An Integrative Approach for Tagging Genes, Gene Families, and Protein Domains BioMed Research International Article ID 918710

[14] Miyao Y, Sagae K, Saetre R, Matsuzaki T, Tsujii J (2009) Evaluating contributions of natural language parsers to protein-protein interaction extraction. Bioinformatics 25: $394-$ 400.

[15] Krallinger M, Leitner F, Rodriguez-Penagos C, Valencia A (2008) Overview of the protein-protein interaction annotation extraction task of BioCreative II. Genome Biol 9: S4. 
[16] Chih-Hsuan Wei, Bethany R. Harris, Hung-Yu Kao, Zhiyong Lu (2013) tmVar: a text mining approach for extracting sequence variants in biomedical literature, Bioinformatics, Volume 29, Issue 11, Pages 1433-1439

[17] Ding J, Berleant D, Nettleton D, Wurtele E. Mining MEDLINE: abstracts, sentences, or phrases. In: Proceedings of the Pacific symposium on biocomputing, Hawaii, USA, 2002. p. 326-37.

[18] Hao Y, Zhu X, Huang M, Li M (2005) Discovering patterns to extract protein- protein interactions from the literature: Part II. Bioinformatics 21: 3294-3300.

[19] Jenssen,T.K. et al. (2001) A literature network of human genes for high-throughput analysis of gene expression. Nat. Genet., 28, 21-28.

[20] Giuliano C, Lavelli A, Romano L (2006) Exploiting Shallow Linguistic Information for Relation Extraction From Biomedical Literature. Proceedings of the 11th Conference of the European Chapter of the Association for Computational Linguistics.

[21] Van Landeghem S, Saeys Y, Peer Y Van de, De Baets B (2008) Extracting ProteinProtein Interactions from Text using Rich Feature Vectors and Feature Selection. Proceedings of the Third International Symposium on Semantic Mining in Biomedicine 7784.

[22] Yifan Peng, Zhiyong Lu (2017) Deeping learning for extracting protein-protein interactions from biomedical literature. Proceedings of the 2017 Workshop on Biomedical Natural Language Processing

[23] Pyysalo S, Airola A, Heimonen J, Bjo r̈ne J, Ginter F, et al. (2008) Comparative analysis of five protein-protein interaction corpora. BMC Bioinformatics 9 Suppl 3: S6. 
[24] Kabiljo R, Clegg A, Shepherd A (2009) A realistic assessment of methods for extracting gene/protein interactions from free text. BMC Bioinformatics 10: 233.

[25] Blaschke Christian, Valencia Alfonso (2002) The frame-based module of the SUISEKI information extraction system. IEEE Intel Syst 17(2):14-20.

[26] Ono Toshihide, Hishigaki Haretsugu, Tanigam Akira, Takagi Toshihisa (2001) Automated extraction of information on protein-protein interactions from the biological literature. Bioinformatics 17(2):155-61.

[27] Hunter L, Lu Z, Firby J, Jr. WAB, Johnson HL, et al. (2008) OpenDMAP: An open source, ontology-driven concept analysis engine, with applications to capturing knowledge regarding protein transport, protein interactions and cell- type-specific gene expression. BMC Bioinformatics 9: 78 .

[28] Hao Y, Zhu X, Huang M, Li M (2005) Discovering patterns to extract protein- protein interactions from the literature: Part II. Bioinformatics 21: 3294-3300.

[29] Hakenberg J, Plake C, Royer L, Strobelt H, Leser U, et al. (2008) Gene mention normalization and interaction extraction with context models and sentence motifs. Genome Biol 9: S14.

[30] Mark Craven, Johan Kumlien (1999) Constructing biological knowledge bases by extracting information from text sources. In: Proceedings of the 7th International conference on intelligent systems for molecular biology. Heidelberg, Germany, p. 77-86.

[31] Bui, Q.- C., Katrenko, S., \& Sloot, P. M. A. (2010). A hybrid approach to extract 
protein-protein interactions. Bioinformatics, 27(2), 259-265.

[32] Hong woo Chun, Yoshimasa Tsuruoka, Jin-Dong Kim, Rie Shiba, Naoki Nagata, Teruyoshi Hishiki, Jun'ichi Tsujii(2006) Extraction of gene- disease relations from MedLine using domain dictionaries and machine learning. In: The Pacific symposium on biocomputing (PSB), p. 4-15.

[33] Hirschman Lynette, Yeh Alexander, Blaschke Christian, Valencia Alfonso (2004)Overview of BioCreAtIvE: critical assessment of information extraction for biology. BMC Bioinformatics 6(Suppl 1).

[34] Airola A, Pyysalo S, Bjo rne J, Pahikkala T, Ginter F, et al. (2008) All-paths graph kernel for protein-protein interaction extraction with evaluation of cross-corpus learning. BMC Bioinformatics 9: S2.

[35] Bunescu R, Mooney R (2006) Subsequence kernels for relation extraction. In: Weiss Y, Scho 1kopf B, Platt J, eds. Advances in Neural Information Processing Systems 18. CambridgeMA: MIT Press. pp 171-178.

[36] Kim S, Yoon J, Yang J (2008) Kernel approaches for genic interaction extraction. Bioinformatics 24: 118-126.

[37] Domonkos Tikk, Ille's Solt, Philippe Thomas, and Ulf Leser (2013) A detailed error analysis of 13 kernel methods for protein-protein interaction extraction. $B M C$ Bioinformatics 14(1):12.

[38] Vishwanathan SVN, Smola AJ (2002) Fast kernels for string and tree matching. In: 
Proc. of Neural Information Processing Systems (NIPS'02). Vancouver, BC, Canada, pp 569-576.

[39] Zhehuan Zhao, Zhihao Yang, Hongfei Lin, Jian Wang, and Song Gao. (2016) A protein- protein interaction extraction approach based on deep neural network. International Journal of Data Mining and Bioinformatics 15(2):145-164.

[40] Daojian Zeng, Kang Liu, Siwei Lai, Guangyou Zhou, Jun Zhao, et al (2014) Relation classification via Convolutional Deep Neural Network. In Proceedings of the 25th International Conference on Computational Linguistics (COLING). pages 2335- 2344.

[41] Sampo Pyysalo, Filip Ginter, Hans Moen, Tapio Salakoski, and Sophia Ananiadou(2013) Distributional semantics resources for biomedical text pro- cessing. In International Symposium on Languages in Biology and Medicine (LBM). pages 39-44.

[42] Rezarta Islamaj Dogan, Sun Kim, Andrew Chartr-aryamontri, Chih-Hsuan Wei, Donald C. Comeau, and Zhiyong (2017) Overview of the BioCreative VI Precision Medicine Track In Proceddings of the BioCreative VI Workshop. page 83-87

[43] Qingyu Chen, Nagesh C.Panyam, Aparna Elangovan, Melissa Davis, Karin Verspoor (2017) Document Triage and Relation Exgtraction for Protein-Protein interactions affected by Mutation. In Proceddings of the BioCreative VI Workshop. page 102-105

[44] Tung Tran, Ramakanth Kavuluru (2017) Exploring a Deep Learning Pipeline for the BioCreative VI Precision Medicine Task. In Proceddings of the BioCreative VI Workshop. page 106-109 
[45] Panyam NC, Verspoor K, Cohn T, Ramamohanarao K. Biomedical Relation

Extraction (2016). ASM Kernel: Graph Kernel using Approximate Subgraph Matching for Relation Extraction. Australasian Language Technology Association Workshop.

[46]https://www.ncbi.nlm.nih.gov/CBBresearch/Lu/Demo/PubTator/index.cgi?user=bc6pp im\&searchtype $=$ PubMed_Search\&query $=$ TrainingSet $[$ collection $] \&$ page $=1 \& \operatorname{tax}=\#$ T 19372 599

[47] Pyysalo S, Ginter F, Heimonen J, Björne J, Boberg J, Järvinen J, Salako- ski T (2007) BioInfer: A Corpus for Information Extraction in the Biomedical Domain. $B M C$ Bioinformatics, 8(50)

[48] Bunescu R, Ge R, Kate RJ, Marcotte EM, Mooney RJ, Ramani AK, Wong YW (2005)Comparative Experiments on Learning Information Extractors for Proteins and their Interactions. Artif Intell Med, Summarization and Information Extraction from Medical Documents 33(2):139-155.

[49] Chowdhary,R. et al. (2009) Bayesian inference of protein-protein interactions from biological literature. Bioinformatics, 25, 1536-1542.

[50] Fundel,K. et al. (2007) RelEx - Relation extraction using dependency parse trees. Bioinformatics, 23, 365-371.

[51] Rinaldi,F. et al. (2010) OntoGene in BioCreative II.5. IEEE/ACM Trans. Comput. Biol. Bioinformatics, 7, 472-480.

[52] Marie-Catherine De Marneffe, Timothy Dozat, Na- talia Silveira, Katri Haverinen, 
Filip Ginter, Joakim Nivre, and Christopher D Manning (2014) Univer- sal Stanford dependencies: A cross-linguistic typology. In Proceedings of 9th International Conference on Language Resources and Evaluation (LREC). volume 14, pages 4585-4592. 